\title{
TECLADO CHIBCHA: UN SOFTWARE LINGÜÍSTICO PARA LOS SISTEMAS DE ESCRITURA DE LAS LENGUAS BRIBRI Y CABÉCAR
}

\author{
Sofía Flores Solórzano
}

\begin{abstract}
RESUMEN
El proyecto Teclado Chibcha surgió a raíz de las dificultades técnicas con las que se enfrentan los indígenas costarricenses y las personas interesadas en el estudio de sus lenguas para escribir en medios digitales. La aplicación diseñada permite el apilamiento de los diacríticos que emplean los alfabetos prácticos de las lenguas bribri y cabécar, ello gracias a la utilización de la fuente libre Charis SIL y un mapa del teclado que facilita la introducción de estos caracteres en el sistema operativo Windows. El resultado es una herramienta de fácil instalación que brinda una solución a un problema técnico que tiene al mismo tiempo profundas implicaciones educativas y culturales. Palabras clave: Unicode, escritura, mapa del teclado, diacríticos, educación indígena.
\end{abstract}

\begin{abstract}
The project Teclado Chibcha was designed to overcome the technical difficulties that face the indigenous people from Costa Rica, and in general the people interested in the study of their native languages, to write in digital media. The application allows stacking the diacritics used by the practical alphabets of the bribri and cabécar languages; all this thanks to the open font Charis SIL and a keyboard layout to facilitate the introduction of these characters in the operating system Windows. The result is a tool of easy installation that solves a technical problem with deep cultural and educational implications.
\end{abstract}

Key words: Unicode, writing, keyboard layout, diacritic, indigenous education.

\section{Introducción}

El problema actual al que se enfrentan aquellas personas que quieren escribir en las lenguas bribri y cabécar es la dificultad para introducir varios diacríticos en una misma grafía, es decir, apilar varias marcas suprasegmentales en una misma letra , ej. à̀, ã̀, è̀, é, ộ. Todos los

M.Sc. Sofía Flores Solórzano. Profesora Interina. Departamento de Comunicación y Lenguaje, Escuela de Estudios Generales. Universidad de Costa Rica.

Correo electrónico: sofia.flores@ucr.ac.cr

Recepción: 31 05- 2011

Aceptación: 14- 06- 2011 
grafemas que usan estas lenguas existen en Unicode ${ }^{1}$, pero no siempre es posible introducirlos fácilmente desde el teclado de la computadora, especialmente las marcas diacríticas, y conseguir una óptima representación de los caracteres tanto en la pantalla como en la impresión. La situación se agrava cuando, ante la dificultad de introducir ciertas combinaciones, se tiende sencillamente a suprimirlas. Ello conduce a una confusión que afecta sobre todo al sistema de educación bilingüe que se imparte en las escuelas de las reservas indígenas de Costa Rica desde los años noventa.

Recordemos que la escolarización de bribris y cabécares comienza en la segunda mitad del siglo XX con la creación de las primeras escuelas en la Reserva Indígena de Talamanca, provincia de Limón (en 1964, la Misión Paulina funda la primera escuela en Amubre y en 1970, el Ministerio de Educación inaugura las escuelas de Bambú, Katsi y Chiroles). El enfoque en esta primera etapa es monolingüe, se enseña a los niños indígenas a leer y escribir en castellano. Poco a poco, esa tendencia cambia, se crea la Asesoría Nacional de Indigenismo en 1985 y posteriormente en 1994 el Departamento de Educación Indígena del Ministerio de Educación. En consecuencia, se intenta que la educación en los territorios indígenas adquiera un enfoque intercultural bilingüe y se fomenta la enseñanza de las lenguas autóctonas, concretamente a través de las clases de lengua y cultura, por eso, a partir de 1996, varios lingüistas de la Universidad de Costa Rica y la Universidad Nacional ofrecen talleres sobre la escritura en estas lenguas a maestros que hablan la lengua indígena, pero que no la escribían (Lamounier 2009).

Es importante reseñar que ninguna de las lenguas indígenas de Costa Rica contaba originalmente con un sistema de escritura autóctono. En la década de 1970, Jack Wilson y Adolfo Constenla elaboran un alfabeto práctico bribri teniendo en cuenta usos previos y usando los caracteres que disponía una máquina de escribir de la época. Posteriormente, con base en este alfabeto, Margery Peña (1986) diseña un alfabeto práctico cabécar.

Estos alfabetos prácticos se basan en el alfabeto en español y no en el Alfabeto Fonético Internacional (AFI o IPA por sus siglas en inglés) y se han usado en todos los textos didácticos publicados por el Departamento de Educación Indígena del Ministerio de Educación de Costa Rica y en prácticamente toda la investigación reciente. Presentan, en ambas lenguas, las siguientes grafías: a, a , b, ch, d, e, ë, e, i, $\underline{i}, j, k, 1, m, n, \tilde{n}, o, \ddot{o}, p$,

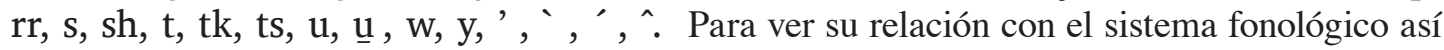
como la historia y los criterios empleados en la elaboración, véase Wilson (1982), Constenla et al. (1998) y Margery Peña (1989).

Anteriormente, para escribir directamente desde el teclado de la computadora en bribri o en cabécar en un sistema operativo Windows y en un procesador de textos WYSIWYG (What you see is what you get), el usuario debía, en primer lugar, instalar una fuente apropiada para la representación del apilamiento de diacríticos y, en segundo lugar, utilizar un programa de reconfiguración del teclado (KeyMan, MSKLC, entre otros), así como modificar el comportamiento de cada tecla para que genere un carácter distinto, por ejemplo, un diacrítico combinable. También, se podía optar por el software propietario LaserYukon, el cual brinda soporte para las lenguas Athapaskan, así como para varias lenguas africanas y americanas. LaserYukon tiene una fuente propia y un mapa del teclado, pero su principal desventaja es que la fuente al ser cerrada y de licencia muy restrictiva no se puede modificar ni incrustar en un documento PDF, ni en ninguna publicación.

En el año 2008, gracias a la financiación de la Secretaría de la Unesco de Costa Rica y la asesoría del Dr. Adolfo Constenla Umaña, desarrollamos el Teclado Chibcha, un software 
específico para la escritura del bribri y el cabécar que se distribuye sin ninguna restricción y de manera gratuita desde el servidor del Instituto de Investigaciones Linguísticas de la Universidad de Costa Rica ${ }^{2}$. Durante estos tres últimos años, hemos brindado soporte web a los usuarios en la instalación y uso de este software; también, hemos añadido compatibilidad con las últimas versiones de Windows (Vista y Windows 7).

\section{Metodología}

El software que presentamos no ha sido un desarrollo desde cero, pues se ha contado con una serie de recursos disponibles en Internet que han posibilitado esta tarea. A continuación, se enumeran los pasos seguidos.

\subsection{Selección de una fuente Unicode que permita el apilamiento de varios diacríticos}

En la versión 1.0 del Teclado Chibcha, se optó por la fuente Doulos SIL. Esta es una fuente no propietaria, desarrollada por El Instituto Lingüístico de Verano (Summer Institute of Linguistics, $I n c^{3}$ ), que usa una licencia abierta, lo cual permite su distribución y modificación, así como su utilización en otro software, siempre y cuando se mantenga intacta la licencia. Doulos SIL es un tipo de letra con serifa, muy similar a Times, que combina de manera óptima las marcas suprasegmentales. Sin embargo, no es una fuente muy apropiada para la impresión profesional, por eso, en la versión 2.0 del Teclado Chibcha, la sustituimos por otra fuente no propietaria, Charis $S I L^{4}$, desarrollada también por el Instituto Lingüístico de Verano.

Charis SIL es un tipo de letra con serifa muy similar a Bitstream Charter, una de las primeras fuentes diseñadas específicamente para las impresoras láser. Al igual que Doulos combina de manera óptima las marcas suprasegmentales, además de proporcionar muchos otros caracteres y símbolos útiles para los lingüistas. A diferencia de Doulos, ofrece una tipografía mucho más profesional en los documentos impresos y tiene varios estilos: regular, cursiva, negrita y cursiva negrita.

\subsection{Configuración del mapa del teclado (keyboard layout)}

Para crear una nueva disposición del teclado o un mapa del teclado que permita la introducción de los caracteres Unicode, especialmente las marcas diacríticas combinables, utilizamos la aplicación Microsoft Keyboard Layout Creator (MSKLC) $)^{5}$. MSKLC es un recurso que cualquier usuario del sistema operativo Windows puede obtener e instalar. Permite definir una nueva disposición del teclado para cualquier idioma que Windows no soporte y además, modificar un mapa del teclado existente, así como construir las librerías necesarias para instalar el nuevo teclado en otros equipos y en distintas arquitecturas. El diseño del Teclado Chibcha (Figura 1) se basa en el teclado del español de Costa Rica, facilitando que el usuario familiarizado con el teclado en español pueda localizar fácilmente las grafías especiales de las lenguas indígenas.

\subsection{Empaquetado}

Con el programa Inno Setup ${ }^{6}$, se empaquetan los archivos y carpetas generados con MSKLC (*.klc, *.msi, *.dll), junto con la fuente Charis SIL y la documentación pertinente 
(instrucciones, licencia...). Este último paso solo es necesario para facilitar la instalación del nuevo mapa del teclado y la fuente en otros equipos.

\section{Resultados}

El producto final es un software para la escritura de las lenguas bribri y cabécar en sus alfabetos prácticos, aunque también se incluye, en la última actualización del 2011, varias grafías IPA útiles para la transcripción fonética de las seis lenguas indígenas que se hablan actualmente en Costa Rica. La aplicación consta de dos partes: la fuente Charis SIL y un mapa del teclado (Figura 1).

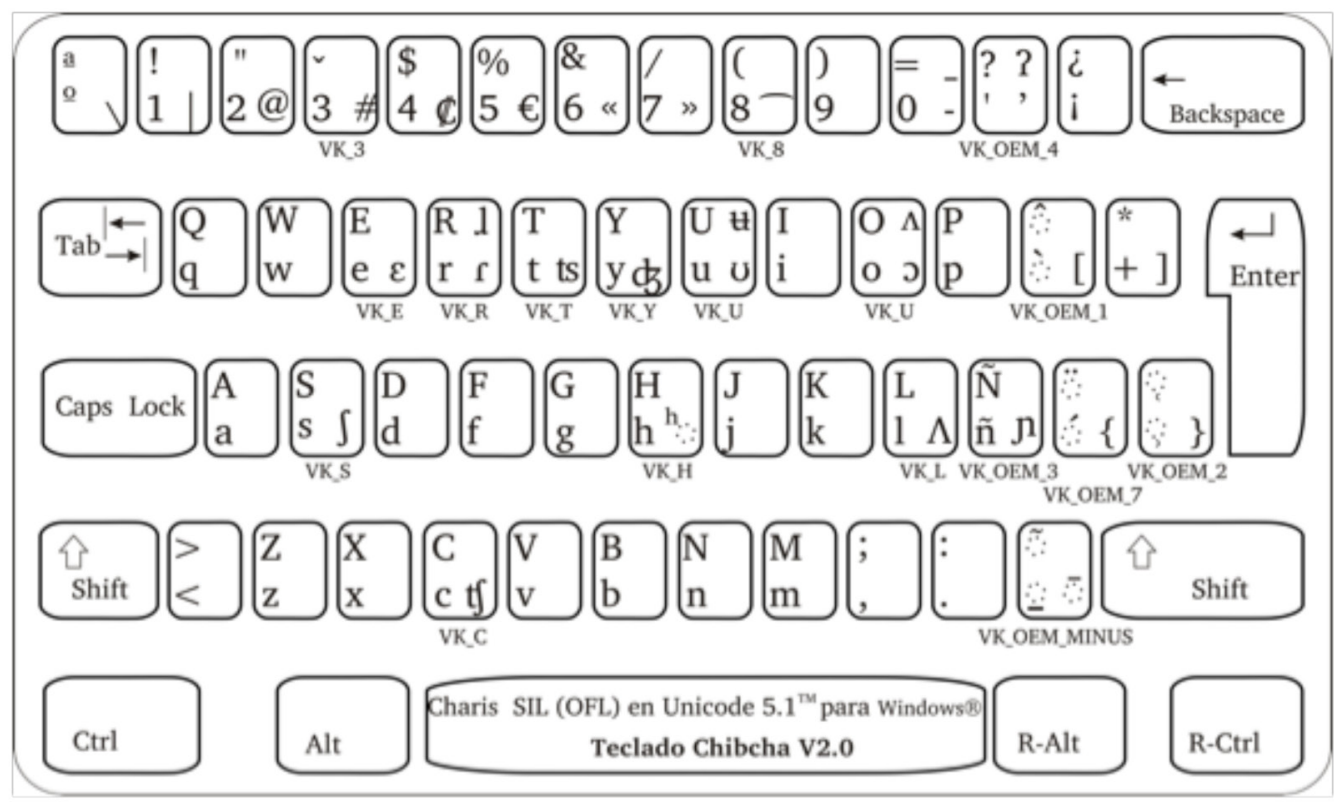

Figura 1. Disposición de los caracteres en El Teclado Chibcha. Fuente: elaboración propia

Es importante tener en cuenta que para poder usar esta herramienta se debe seleccionar el Teclado Chibcha en la barra de idiomas y la fuente Charis SIL en el editor de textos. En el Cuadro 1, puede verse en detalle los diacríticos que se pueden insertar desde el teclado mediante las siguientes combinaciones de teclas:

a) Para colocar diacríticos (tildes, guiones), se digita primero la letra y a continuación los diacríticos que se quieren insertar; se pueden apilar varios diacríticos en una misma letra.

b) Las grafías (letras o diacríticos) ubicadas en la parte superior de las teclas se obtienen digitando la tecla la mayúscula (Shift) y la tecla que tiene la grafía.

c) Las grafías (letras o diacríticos) ubicadas en la parte inferior derecha se obtienen digitando la tecla ALTGR y la tecla de la grafía.

d) Las grafías (letras o diacríticos) ubicadas en la parte superior derecha se obtienen mediante la combinación AltGR + Shift + la tecla que tiene la grafía. 
Cuadro 1. Marcas Diacríticas Combinables

\begin{tabular}{|c|l|l|l|}
\hline Grafía & Código Unicode & Descripción & Teclas \\
\hline$\sim$ & U+0300 & Acento grave combinable & V_OEM_1 \\
\hline$\cdot$ & U+0301 & Acento agudo combinable & VK_OEM_7 \\
\hline$\sim$ & U+0302 & Acento circunflejo combinable & VK_OEM_1 + Mayúscula \\
\hline$\cdot$ & U+0308 & Diéresis combinable & VK_OEM_7 + Mayúscula \\
\hline- & U+0331 & Acento largo inferior combinable & VK_OEM_MINUS \\
\hline$\sim$ & U+0303 & Tilde combinable & Mayúscula + VK_OEM_MINUS \\
\hline- & U+0304 & Acento largo combinable & AltGr + VK_OEM_MINUS \\
\hline$\sim$ & U+030C & Acento anti-circunflejo combinable & Shift + VK_3 \\
\hline O & U+0339 & Semicírculo inferior derecho combinable & VK_OEM_2 \\
\hline o & U+031C & Semicírculo inferior izquierdo combinable & Shift + VK_OEM_2 \\
\hline
\end{tabular}

\section{Discusión}

Según la clasificación de los estados de conservación de las lenguas indígenas de Bauman (1980), tanto el bribri (6.000 hablantes) como el cabécar (8.500 hablantes) se encuentran entre el estado de resistencia y declinación dependiendo de la comunidad. Así, las comunidades que se asientan en zonas más montañosas y de difícil acceso están en estado de resistencia; mientras que las comunidades que viven en zonas bajas y cerca de poblaciones no indígenas en estado de declinación (Constenla Umaña 2011). La pérdida de hablantes se produce como consecuencia de un proceso complejo de asimilación del castellano como lengua y cultura dominante, determinado por las desventajas sociales y económicas que conlleva el pertenecer a una minoría indígena. La transmisión de la lengua autóctona en el hogar falla debido a que en muchos casos los padres ya no hablan la lengua indígena o la asocian con la ausencia de oportunidades; por eso, prefieren que sus hijos aprendan la lengua de prestigio (Adelaar 2007).

A pesar de que se han hecho esfuerzos a favor de la educación bilingüe en las escuelas indígenas, hace falta una política lingüística más decidida para rescatar a estas lenguas de su estado de resistencia y declinación. El objetivo de este proyecto ha sido promover la comunicación y la producción textual en las lenguas bribri y cabécar en medios digitales, especialmente, entre estudiantes, maestros y jóvenes indígenas, a través de una aplicación que facilite la escritura en los alfabetos prácticos de estas lenguas. El resultado, Teclado Chibcha, es un software gratuito especialmente diseñado para sistemas de escritura que requieren de una fuente o tipo de letra que apile adecuadamente varios diacríticos.

La respuesta en estos tres años ha sido heterogénea. Estudiantes e investigadores de la Universidad de Costa Rica vinculados a la Facultad de Letras así como funcionarios del Departamento de Educación Indígena del Ministerio de Educación de Costa Rica utilizan el Teclado Chibcha; sin embargo, no existe constancia de un uso difundido en las comunidades indígenas. Aparentemente, la difusión exclusiva a través del sitio web es insuficiente, pues 
aunque hay computadoras en algunas escuelas indígenas, y algunos maestros cuentan con una propia, no siempre hay acceso a Internet y tampoco, los maestros han recibido asesoría del Ministerio de Educación.

Finalmente, debemos mencionar que la ortografía bribri y cabécar contrasta con algunos sistemas empleados por otras comunidades indígenas de Centroamérica, por ejemplo, en Guatemala, los mayas ha luchado por el reconocimiento oficial de su propia ortografía; en Chiapas, los educadores mayas han elegido un sistema de escritura no basado en el español. No obstante, en Yucatán, la larga tradición de alfabetización en maya yucateco ha hecho que se preserve la ortografía que se ha empleado desde que comenzó la alfabetización (Maxwell 2006). ¿Cuál es la ortografía que deben emplear los bribris y cabécares? Esta es una pregunta que deben contestar ellos mismos, nosotros solo ponemos a su disposición una herramienta que en ningún momento intenta ser normativa en cuanto a la ortografía que debe emplearse.

Nuestro deseo es que independientemente de la ortografía que se escoja, con este software u otros similares, se incremente en el mediano y largo plazo el número de textos producidos por los indígenas costarricenses en sus lenguas autóctonas a través de diferentes aplicaciones informáticas, principalmente procesadores de texto, pero también clientes de mensajería instantánea (chat), servicios de correo web, diseño gráfico, y en general, todas aquellas aplicaciones que implementen el sistema Unicode.

\section{Notas}

1. Unicode es un estándar universal de codificación para el intercambio de los caracteres empleados en la mayoría de los sistemas de escritura del mundo, la versión 6.0 tiene más de un millón de caracteres. El consorcio Unicode está integrado por representantes de las principales compañías de la industria de software y hardware, quienes desarrollaron un sistema para intercambio de los caracteres a través de los sistemas informáticos, los caracteres unicode tiene un código único y se pueden interpretar en 32 bits (UTF-23), 16 bits (UTF-16) u 8 bits (UTF-8) (The Unicode Consortium, 2011).

2. http://inil.ucr.ac.cr/teclado_chibcha

3. http://www.sil.org/

4. http://scripts.sil.org/cms/scripts/page.php?item_id=CharisSIL_Technical

5. http://msdn.microsoft.com/en-us/goglobal/bb964665.aspx

6. http://www.jrsoftware.org/ishelp/

\section{Bibliografía}

Adelaar, Willen. 2007. “Latin America”. En: Moseley (ed.), 97-103.

Bauman, James. 1980. A Guide to Issues in Indian Language Retention. Washington D.C.: Center for applied linguistics. 
Brown, Keith y Sara Ogilvie (eds.). 2009. Concise Encyclopedia of Languages of the World. Oxford: Elsevier.

Constenla, Adolfo, Feliciano Elizondo y Francisco Pereira. 1998. Curso básico de bribri. San José: Editorial de la Universidad de Costa Rica.

Constenla Umaña, Adolfo. 2006. “Chibchan”. En: Brown y Ogilvie (eds.) “Concise Encyclopedia of Languages of the World”, 208-210.

Constenla Umaña, Adolfo. En prensa. "Estado de conservación y documentación de las lenguas de América Central pertenecientes a las agrupaciones jicaque, lenca, misumalpa, chibchense y chocó".

Lamounier, Alice. 2009. "La enseñanza del cosmos: la experiencia de los maestros de cultura bribris y cabécares". InterCAmbio 6 (7): 31-54.

Margery Peña, Enrique. 1986. Abecedario ilustrado cabécar. San José: Ministerio de Educación Pública.

1989. Diccionario cabécar - español, español - cabécar. San José: Editorial Universidad de Costa Rica.

Maxwell, J.M. 2006. “Mayan Languages”. En: Brown y Ogilvie (eds.), 705-709.

Moseley, Christopher (ed.). 2007. Encyclopedia of the World's Endangered Languages. New York: Routledge.

The Unicode Consortium. 2011. The Unicode Standard, Version 6.0.0. Mountain View, CA: The Unicode Consortium.

Wilson, Jack L. 1982. “El alfabeto bribri”. En: “Diccionario fraseológico bribri-español, español-bribri”, 15-24. 
